\title{
Putting Sarcasm Detection into Context: The Effects of Class Imbalance and Manual Labelling on Supervised Machine Classification of Twitter Conversations
}

\author{
Gavin Abercrombie and Dirk Hovy \\ Center for Language Technology \\ University of Copenhagen, Denmark \\ Njalsgade 140, DK-2300 Copenhagen S \\ jst662@alumni.ku.dk, dirk.hovy@hum.ku.dk
}

\begin{abstract}
Sarcasm can radically alter or invert a phrase's meaning. Sarcasm detection can therefore help improve natural language processing (NLP) tasks. The majority of prior research has modeled sarcasm detection as classification, with two important limitations: 1. Balanced datasets, when sarcasm is actually rather rare. 2. Using Twitter users' self-declarations in the form of hashtags to label data, when sarcasm can take many forms. To address these issues, we create an unbalanced corpus of manually annotated Twitter conversations. We compare human and machine ability to recognize sarcasm on this data under varying amounts of context. Our results indicate that both class imbalance and labelling method affect performance, and should both be considered when designing automatic sarcasm detection systems. We conclude that for progress to be made in real-world sarcasm detection, we will require a new class labelling scheme that is able to access the 'common ground' held between conversational parties.
\end{abstract}

\section{Introduction}

Sarcasm, or verbal irony, is prevalent both in spoken and written communication, and can radically alter or invert a phrase's meaning. Automatic sarcasm detection can therefore help improve natural language processing (NLP) tasks, such as sentiment analysis, where failure to take ironic intent into account has been recognised as a major cause of errors.

However, automatic sarcasm detection is a nontrivial problem, and research into this subject is in its infancy. The majority of prior research has treated sarcasm detection as a classification task, with two important limitations: 1. It focuses on balanced datasets, when sarcasm is actually rather rare. 2. In order to obtain labelled data for supervised learning, many studies relied on Twitter users' supposed self-declarations of sarcasm in the form of hashtags such as \# sarcasm, but sarcasm can take many forms.

Although reporting impressive results for sarcasm detection, even state-of-the-art systems fail to address the above issues. Research suggesting that verbal irony occurs in less than a fifth of conversations (Gibbs, 2000) implies that, rather than using balanced datasets, a more realistic approach may be to view sarcasm recognition as a problem of anomaly detection, in which positive examples are scarce. While convenient, obtaining labelled data from hashtags has been found to introduce both noise, in the form of incorrectly labelled examples, and bias to the datasets used analysis suggests that only certain forms of sarcasm are likely to be tagged in this way (Davidov et al., 2010), and predominantly by certain types of Twitter users (Bamman and Smith, 2015).

To address these issues, we create a novel corpus of manually annotated Twitter conversations and, using the feature classes of Bamman and Smith (2015), perform sarcasm classification experiments on both balanced and unbalanced datasets. We also compare model performance to a dataset of conversations automatically retrieved using hashtags.

Our contributions In this paper, we present a novel corpus of manually annotated two-part Twitter conversations for use in supervised classification of sarcastic and non-sarcastic text. We compare human vs. machine learning classification performance under varying amounts of contextual information, and evaluate machine perfor- 
mance on balanced and unbalanced, and manually labelled and automatically retrieved datasets.

\section{Data}

Most prior research into sarcasm detection has been conducted on Twitter. To make comparisons with other research, and because use of sarcasm seems to be prevalent on Twitter, we too make use of Twitter data for this study.

However, the collection of data using explicit markers of sarcasm (hashtags) has been shown to introduce bias to the datasets used in prior research (Davidov et al., 2010; González-Ibánez et al., 2011; Maynard and Greenwood, 2014; Bamman and Smith, 2015). We therefore create a novel hand-annotated corpus of contextualised sarcastic and non-sarcastic Twitter conversations. For comparison, we also create an automatically collected dataset using hashtags.

Corpus creation The data set is taken from a Twitter corpus of 64 million tweets gathered in 2013. Matching tweet reply IDs to the status IDs of other tweets, and filtering by language, produces 650,212 two-line English Twitter 'conversations.' We manually annotate these, finding 448 positive examples, to which we add 1,792 negatively labelled examples in which sarcasm was found not to be present. The resulting corpus contains 2,240 conversations in total. A second corpus, which is automatically retrieved using hashtags, is created, producing 448 Twitter conversation where the second tweet contains \# sarcasm, and 1,792 without this feature. Following previous work, we remove usernames and web addresses. For the second corpus, we also remove the term \# sarcasm. We collect up to 3,200 historical tweets written by each user ID in the datasets.

Annotation We annotated the conversations manually with full access to the text of the conversations and user profile information and tweet history of the users. Following prior work (Kreuz and Caucci, 2007), and because people have been found to conflate many forms of verbal irony under the term sarcasm (Gibbs, 1986), positive labels were not assigned according to any fixed criteria or definition, but according to our intuitive understanding of whether or not examples contained verbal irony ${ }^{1}$

\footnotetext{
${ }^{1}$ This was also necessary because prior sarcasm detection studies relied on self-annotation of sarcasm by Twitter users applying their own judgements of sarcastic mean-
}

\section{Human performance baseline study}

This study was undertaken with the participation of 60 native English speaking volunteers. We randomly selected 300 Twitter conversations from the corpus and assigned them each one of five conditions: tweet only - the text of the reply tweet from the conversation, tweet + author - including access to the Twitter profile of the author, tweet + audience - including the profile of the writer of the original tweet in the conversation, tweet + environment - the texts of both tweets, and tweet + author + audience + environment - access to all the above information. Each participant rated 10 conversations.

Procedure We asked two participants to rate the reply tweet of each conversation as either sarcastic or non-sarcastic. Again, following Kreuz and Caucci (2007), raters were not provided with a definition of sarcasm, but were asked to judge the tweets based on their intuitive understanding of the term.

Inter-rater agreement We use inter-rater agreement measures to assess both the difficulty of the sarcasm recognition task under different conditions and the reliability of the participants. We report both raw percentage agreement and - as in previous work on sarcasm annotation (Swanson et al., 2014) - Krippendorffs $\alpha$, which takes into account expected chance disagreement.

Contrary to expectations, annotators are not more likely to agree if given access to more information. Agreement is highest for the tweet only condition $(\%=70.49, \alpha=0.35)$. Krippendorffs $\alpha$ scores for tweet + audience $(0.08)$ and tweet + original + author + audience $(0.18)$ are very low, while tweet + audience produces a negative score $(-0.10)$ which indicates that agreement is below chance levels.

Rater reliability Agreement scores are generally low. Only two pairs obtain 'good' agreement scores. ${ }^{2}$ The majority (20 pairs) receive a score between 0.0 and 0.67 , while eight of the pairs achieve negative scores, indicating less than chance expected agreement. Two possible explanations for low rater agreement are (1) that sarcasm recognition is a difficult task for humans (Kreuz and Caucci, 2007; González-Ibánez et al., ing e.g., Davidov et al. (2010) 2010, González-Ibánez et al. (2011).

${ }^{2}$ Krippendorff (2012) considers 0.67 to be lowest acceptable agreement score. 
2011), especially without access to the surrounding context (Filatova, 2012; Wallace et al., 2014), and (2) that people undertaking such tasks remotely online are often guilty of 'spamming,' or providing careless or random responses (Hovy et al., 2013).

To mitigate the effects of unreliable raters and get an upper bound for human performance, we use two measures: (1) discard the results of the worst performing rater in each pair (in terms of F1) and use the vote of the higher scoring raters. (2) identify the least trustworthy raters and downweight their votes using scores from an itemresponse model, MACE (Hovy et al., 2013).

The first requires access to the original annotated labels, the latter can be done with or without access to the gold standard. We compare both F1 and Area Under the Curve (AUC) scores of both raters in each pair, the better performing rater only, and the MACE most competent rater in each pair over all conditions.

For both measures, MACE competent rater scores (F1: 0.547; AUC: 0.731) are marginally higher than the mean of both raters (F1: 0.523; AUC: 0.729), while the best rater scores (F1: 0.641; AUC: 0.817) are highest of all, as might be expected.

\subsection{Machine classification experiments}

To compare human to machine performance, we fit binary classification models on both balanced and unbalanced splits of the two datasets.

Experimental setup We evaluate performance using a standard logistic regression model with $\ell_{2}$ regularization, evaluated via five-fold crossvalidation.

Features For the five conditions, we use the following feature classes, as named and described by Bamman and Smith (2015):

Tweet features: Unigrams, bigrams, Brown cluster unigrams, Brown cluster bigrams, part-ofspeech features, pronunciation features, and intensifier features.

Author features: Author historical salient terms, profile information, profile unigrams.

Audience features: Audience historical salient terms, profile unigrams, profile information, and historical communication features.

Environment features: Pairwise Brown features and unigram features of audience tweets.
Normalisation We convert all features to binary or numeric values and normalize them to the range between zero and one.

Procedure Following Bamman and Smith (2015), we evaluate classification performance on the above feature sets in the following combinations: tweet features only, tweet + author features, tweet + audience features, tweet + environment features, and tweet + author + audience + environment.

\section{Results}

Accuracy is commonly reported in classification tasks, but unsuitable for unbalanced datasets (López et al., 2013), so we report two other metrics frequently used with uneven class distributions: F1 score, and Area Under the ROC Curve (AUC), which reflects the relationship between the true positive rate (TPR) and false positive rate (FPR). Unlike accuracy, these measures penalize predicting only the majority class. AUC is considered to be more resistant to the skew of unbalanced data than F1 (Fawcett, 2004).

Comparison with baselines Figure 1 compares random performance, human raters, ${ }^{3}$ and the classifier's AUC scores. The scores of both the human raters and the machine classifier surpass random performance in all conditions, with the classifier attaining the lowest score of 0.615 on tweet $+a u$ thor features. Machine classification is not, however, able to match human performance. But there are parallels between human and machine performance: the classifier achieves its highest score using tweet + environment features (human: 0.802; machine: 0.630). Interestingly, both humans and the classifier appear to suffer from an 'information saturation' effect, obtaining lowest scores when trained on a combination of all the possible features.

Machine classification performance across conditions We have two data-related factors that affect performance, namely (1) label prevalence (i.e., balanced vs. unbalanced splits), and (2) the labelling scheme (manual vs. automatically induced from \#sarcasm). Figure 2 shows the effects on F1 and AUC for each combination of these two factors under all five conditions.

\footnotetext{
${ }^{3}$ Using the MACE most competent rater scores, which we judge to be the fairest comparison.
} 


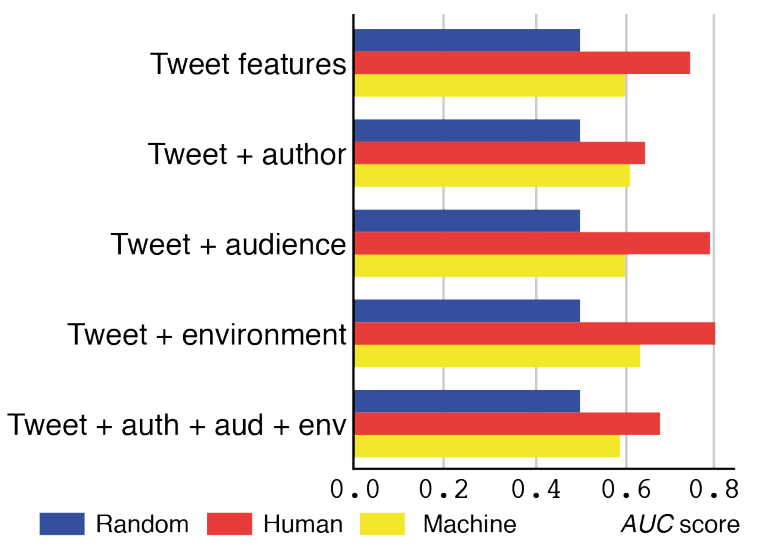

Figure 1: AUC scores of random performance, the most competent human raters, and machine classification on an unbalanced split of the manually annotated data.

\section{Class label balance}

AUC scores are largely unaffected by change in label balance. We see broadly similar results on both balanced and unbalanced data splits across all the feature classes on both corpora. The small changes in performance that do occur can be attributed to the increase in size of the unbalanced datasets, which have more negative training examples compared to the balanced sets.

However, for both corpora, and across all feature classes, F1 scores suffer large drops on the unbalanced data compared to results on the balanced datasets. These results indicate that F1, known to be biased to the negative class and to ignore the effect of true negatives (Powers, 2015), may not be a suitable metric for this task, as it is very sensitive to the changes in class balance of the datasets. Nevertheless, even when measured with AUC score, in the majority of feature configurations classifier performance drops on the unbalanced datasets. Results therefore suggest that class balance (and dataset size) should be taken into account when designing sarcasm detection systems. Labelling scheme

Overall, higher scores are achieved with the automatically collected corpus. All feature combinations obtain higher F1 and AUC scores on this data using the balanced split, as do tweet + auth and tweet + aud on the unbalanced data. This points to greater homogeneity in the data in the automatically collected corpus. This may be because it is often certain types of users, such as those who do not know their audience personally, who feel the need to label their sarcastic statements with hash- tags (Bamman and Smith, 2015). Manually annotated data includes instances of sarcasm which the author has not deemed necessary to explicitly label as sarcastic. This may lead to greater variation in the features of the positive examples in the manually annotated data, and hence lower classification scores.

The only feature category in which $\mathrm{F} 1$ and AUC scores for the manually annotated data are higher than those for the automatically collected data are on the unbalanced split for tweet features (F1: +0.012, AUC: +0.08$)$ and tweet + env (F1: +0.037 , AUC: +0.015$)$, while tweet + auth + aud + env produces a higher F1 score $(+0.275)$, but a slightly lower AUC score (-0.023). These figures point to the fact that for the manually annotated data, performance is best when linguistic features from both tweets in the conversations are included. Indeed, on both balanced and unbalanced data splits of the manually annotated data, better results are generally produced using these textual features than using features related to the writers of those texts. It would therefore seem that the annotation process has introduced some biases to the data. This process, in which sarcasm, or the ambiguous possibility of sarcasm, is first recognised in the dialogues and then confirmed by scrutiny of users Twitter pages, heavily favours textual features. Twitter conversations automatically selected using hashtags on the other hand, are likely to be highly ambiguous once those hashtags are removed and, as discussed above, more likely to be predictable from information in the conversational participants' profile metadata than from linguistic features.

\section{Related Work}

Research in both cognitive psychology (Utsumi, 2000; Gibbs and Colston, 2007) and NLP (Filatova, 2012) has suggested that it may not be possible to produce an overarching definition of sarcasm. Kreuz (1996) noted that use of sarcasm often depends on the 'common ground' people share. Work on human sarcasm recognition (Kreuz and Caucci, 2007) and automatic sarcasm detection (Bamman and Smith, 2015) has relied on people's intuitive understanding of the term 'sarcasm' for rating and data labelling purposes.

Following the insights of Kreuz and Caucci (2007), Carvalho et al. (2009), González-Ibánez et al. (2011) and Tsur et al. (2010), among others, used textual cues for automatic sarcasm detection. 


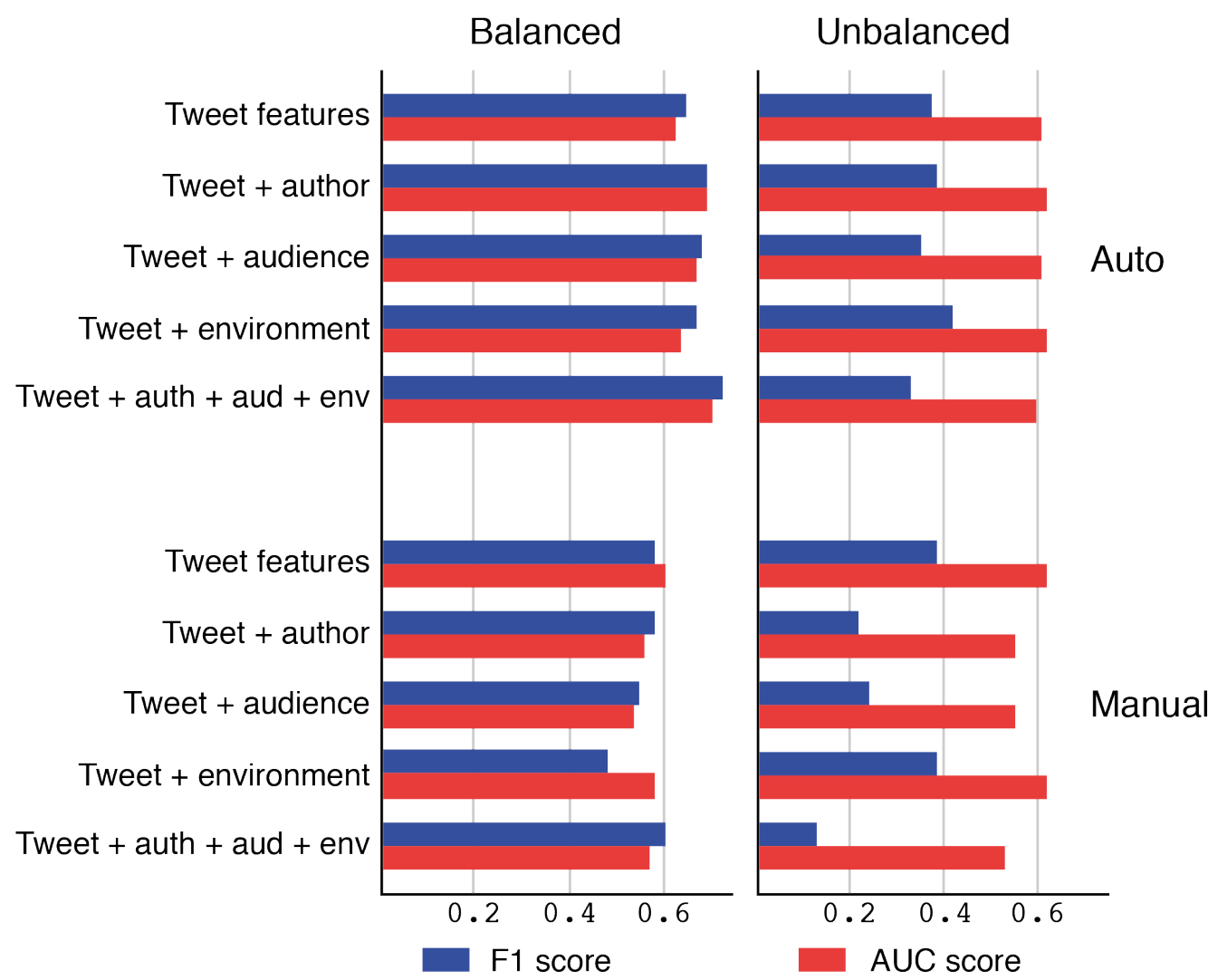

Figure 2: Effects of labeling method (top vs. bottom row) and label prevalance (left vs. right column) on F1 and AUC scores.

Addressing the wider context in which tweets are written, Rajadesingan et al. (2015) mapped information from the posting history of Twitter users to research on why, when, and how sarcasm tends to be used. They also tested their model on both balanced and unbalanced datasets. Bamman and Smith (2015) showed that a variety of contextual features can improve classification performance over use of textual features alone. However, like González-Ibánez et al. (2011) and Maynard and Greenwood (2014), they concluded that the use of hashtags for data labelling introduced biases to their dataset.

\section{Conclusions}

We evaluated the performance of human raters and a machine learning algorithm on sarcasm detection under different information conditions. We find that humans generally benefit from context more than machines, but that machine performance is even more affected by the labeling scheme (automatically induced vs. handannotated) and the prevalence of the target class. Our results indicate that sarcasm detection is far from solved, and that any results on the task need to be viewed in the light of the two factors outlined here.

In automatic sarcasm detection, use of unbalanced datasets led to large drops in F1 scores, due to this metric not taking into account true negatives. As the ratio of TNs is necessarily large for effective sarcasm detection on data in which positive examples are rare, AUC seems a more appropriate performance metric.

Although more robust to class imbalance, AUC scores also varied between the balanced and unbalanced datasets. This indicates that label class balance and dataset size should be taken into account when designing sarcasm detection systems.

Previous work suggests that the automatic selection of positive examples using user-written hashtags biases the data towards (1) particularly ambiguous forms of sarcasm, and (2) 'celebrity' Twitter users who are anxious not to be misunderstood. Our labelling method avoids these pitfalls, as well as eliminating noise in the form of tweets that use sarcastic hashtags but are not in fact ironic. However, in using the labels of an outside observer to the conversations, we may be in- 
troducing other forms of bias. It seems that a gold standard sarcasm corpus would require labelling by the annotators who are party to the 'common ground' shared by the participants in the conversations. It would also need to include those instances that they would not normally publicly mark as being sarcastic with hashtags.

Future work will focus on improving the quality and size of labelled corpora available for this task. It will also explore the use of features from the wider conversational context beyond the twosentence dialogues examined here, and investigate the effects of data labelling method and class balance on media other than Twitter.

\section{Acknowledgements}

We would like to thank the participants who volunteered their time and effort to make this study possible. We also thank the anonymous reviewers for their invaluable comments.

\section{References}

David Bamman and Noah A Smith. 2015. Contextualized sarcasm detection on twitter. In Ninth International AAAI Conference on Web and Social Media.

Paula Carvalho, Luís Sarmento, Mário J Silva, and Eugénio De Oliveira. 2009. Clues for detecting irony in user-generated contents: oh...!! it's so easy;-). In Proceedings of the 1st international CIKM workshop on Topic-sentiment analysis for mass opinion, pages 53-56. ACM.

Dmitry Davidov, Oren Tsur, and Ari Rappoport. 2010. Semi-supervised recognition of sarcastic sentences in twitter and amazon. In Proceedings of the Fourteenth Conference on Computational Natural Language Learning, pages 107-116. Association for Computational Linguistics.

Tom Fawcett. 2004. Roc graphs: Notes and practical considerations for researchers. Machine learning, 31(1):1-38.

Elena Filatova. 2012. Irony and sarcasm: Corpus generation and analysis using crowdsourcing. In $L R E C$, pages 392-398.

Raymond Gibbs and Herbert Colston. 2007. The future of irony studies. Irony in Language and Thought, pages 339-360.

Raymond W Gibbs. 1986. On the psycholinguistics of sarcasm. Journal of Experimental Psychology: General, 115(1):3.

Raymond W Gibbs. 2000. Irony in talk among friends. Metaphor and symbol, 15(1-2):5-27.
Roberto González-Ibánez, Smaranda Muresan, and Nina Wacholder. 2011. Identifying sarcasm in twitter: a closer look. In Proceedings of the 49th Annual Meeting of the Association for Computational Linguistics: Human Language Technologies: short papers-Volume 2, pages 581-586. Association for Computational Linguistics.

Dirk Hovy, Taylor Berg-Kirkpatrick, Ashish Vaswani, and Eduard H Hovy. 2013. Learning whom to trust with mace. In HLT-NAACL, pages 1120-1130.

Roger J Kreuz and Gina M Caucci. 2007. Lexical influences on the perception of sarcasm. In Proceedings of the Workshop on computational approaches to Figurative Language, pages 1-4. Association for Computational Linguistics.

Roger J Kreuz. 1996. The use of verbal irony: Cues and constraints. Metaphor: Implications and applications, pages 23-38.

Klaus Krippendorff. 2012. Content analysis: An introduction to its methodology. Sage.

Victoria López, Alberto Fernández, Salvador García, Vasile Palade, and Francisco Herrera. 2013. An insight into classification with imbalanced data: Empirical results and current trends on using data intrinsic characteristics. Information Sciences, 250:113141.

Diana Maynard and Mark A Greenwood. 2014. Who cares about sarcastic tweets? investigating the impact of sarcasm on sentiment analysis. In Proceedings of LREC.

D. M. W. Powers. 2015. What the F-measure doesn't measure: Features, Flaws, Fallacies and Fixes. ArXiv e-prints, March.

Ashwin Rajadesingan, Reza Zafarani, and Huan Liu. 2015. Sarcasm detection on twitter: A behavioral modeling approach. In Proceedings of the Eighth ACM International Conference on Web Search and Data Mining, pages 97-106. ACM.

Reid Swanson, Stephanie M Lukin, Luke Eisenberg, Thomas Corcoran, and Marilyn A Walker. 2014. Getting reliable annotations for sarcasm in online dialogues. In $L R E C$, pages 4250-4257.

Oren Tsur, Dmitry Davidov, and Ari Rappoport. 2010. Icwsm-a great catchy name: Semi-supervised recognition of sarcastic sentences in online product reviews. In ICWSM.

Akira Utsumi. 2000. Verbal irony as implicit display of ironic environment: Distinguishing ironic utterances from nonirony. Journal of Pragmatics, 32(12):1777-1806.

Byron C Wallace, Laura Kertz Do Kook Choe, and Eugene Charniak. 2014. Humans require context to infer ironic intent (so computers probably do, too). 
In Proceedings of the Annual Meeting of the Association for Computational Linguistics (ACL), pages 512-516. 